\title{
On Structural Optimization of the Propeller Blade
}

\author{
Mikhail Aleshin $^{1 *}$, Aleksandr Smirnov ${ }^{2}$, Margarita Murzina $^{2}$, Yuri Boldyrev ${ }^{3}$ \\ ${ }^{1}$ Lead engineer, Peter the Great St.Petersburg Polytechnic University, Saint-Petersburg, Russia, Polytechnicheskaya, 29, 195251 \\ ${ }^{2}$ Engineer, Peter the Great St.Petersburg Polytechnic University, Saint-Petersburg, Russia, Polytechnicheskaya, 29,195251 \\ ${ }^{3}$ Doctor of technical science, Professor, Peter the Great St.Petersburg Polytechnic University, Saint-Petersburg, Russia, \\ Polytechnicheskaya, 29, 195251 \\ *Corresponding author Email: aleshin@compmechlab.com
}

\begin{abstract}
The results of the structural optimization of propeller blades are presented taking into account its composite structure and pitch change mechanism of the propeller and using FSI (Fluid-Structure Interaction) approaches. The optimality criterion of the problem is the propeller thrust with optimization parameters being the characteristics of the internal structure of the propeller blade made from a composite. Together with the optimization of the blade shape, the problem is considered which concerns the reduction of the deformations caused by loads occurring during the operation of the propeller, since significant deformations of the blades lead to decreased thrust.

Thus, the following optimization problem can be formulated: to find the optimal configuration of the composite material and its microgeometrical parameters along the height of the blade to minimize deformations and increase the thrust of the propeller. At the same time, the optimization parameters are limited by the weight of the propeller and the strength characteristics.

The technique presented in the paper allows us to obtain the reliable values of thrust and reduce the estimated computational time. The influence of the structure of the composite material on the mechanical properties of the blades is shown; the values of deformation of the blades under the action of centrifugal and aerodynamic loads are given.
\end{abstract}

Keywords: optimization, composite materials, blade, aerodynamics, aeroelasticity.

\section{Introduction}

These days, there is an issue in designing propeller blades in a way ensuring the option of changing the basic geometrical parameters of those while they are in motion $[8 ; 9 ; 10]$. This issue arose because the controllable-pitch propellers are not optimal for all phases of operation that units for various purposes go through. Moreover, the air propeller works great at flight speeds that are under the Mach $0.5-0.6[11 ; 12 ; 13]$. With a further increase in the flight speed, air compressibility will occur at the ends of propeller blades, sparking a significant loss of effectiveness. This problem can be solved only by using a propeller with variable geometry, as it allows changing the rotor diameter, the pitch and the twist angle of blades in motion [14; 15].

Recently the numerical experiment used for modeling the spatial turbulent flows behind objects which rotate at a high speed has become more and more popular in aviation and space industry designing. Modeling 3D turbulent flows calls for a lot of computations, but with the development of supercomputers, the complexity level of the problems to be solved keeps on growing. Using modern approaches to numerical modeling and software systems with parallel computing technologies allow the time within which the product is developed and launched to the market to be reduced. An approach where aerodynamic characteristics of propellers are calculated with the use of turbulence models based on the Reynolds-averaged Navier-Stokes equations [1] in a set-up where blade deformations are not considered is incorrect in the general case. Such an approach leads to a significant deviation from the physical reality observed in field studies, which is preconditioned by the lack of consideration of the change in the blade shape caused by aerodynamic loads and vortex formed behind the blades. The blades of modern propellers are made from composites due to the low specific weight of the material and less proneness to wear [2;7], which is important in the design of propellers. Composite materials, among other things, provide a considerable rigidness of the blade structure, which helps to avoid having a lot of change in the blade shape during operation, prevents flutter and the loss of thrust. Aeroelasticity approaches allow us to solve the problem of fluid structure interaction (FSI) of the blade elements with the external distributed loads of approach flow. When such approaches are used, the calculated distributed aerodynamic loads are transferred to the blade deformation analysis modules, which makes it possible to calculate the propeller thrust given the changed shape of the blade. There are two ways of implementing the FSI methods: One-Way FSI and Two-Way FSI.

When problems are solved using the one-way FSI method, with aerodynamic loads being transferred to the module of blade stress-strain analysis (SSA) only once, the deformation of the blade can be estimated, but the aerodynamic characteristics of the blades cannot be recalculated given their changed shape as affected by these loads. This problem can be solved by the second approach - a two way FSI method, where the loads can be transferred both from the aerodynamic module to the SSA module and vice versa. In this approach, when the problem is solved in an unsteady set-up, the so-called vortex approaches [3; 4] can be used Copyright $(\odot) 2018$ Authors. This is an open access article distributed under the Creative Commons Attribution License, which permits unrestricted use, distribution, and reproduction in any medium, provided the original work is properly cited. 
in turbulence modeling such as Scale Adaptive Simulation SST, Detached Eddy Simulation and others. The application of vortex approaches ensures the required precision of the calculation data, since it suggests solving an unsteady three-dimensional problem of propeller rotation in airspace with identification of the most significant vortexes in terms of scale, their interaction with each other and the impact of the pressure pulsations generated by them and exerted on blades. Within such approaches it is possible to obtain aerodynamic loads on the blades which change in time cyclically, and take into account the vortex trail produced by the blades during their rotation.

\section{Problem setting}

The object of the research is a three blade altitude propeller (see Fig. 1). The diameter of the propeller is 2.5 meters. The propeller is isolated, i.e. the propeller rotation is considered without taking into account the wing of the aircraft and the engine pod. The blades of the propeller are made from the composite material Epoxy Carbon Woven Prepreg (230 GPa).

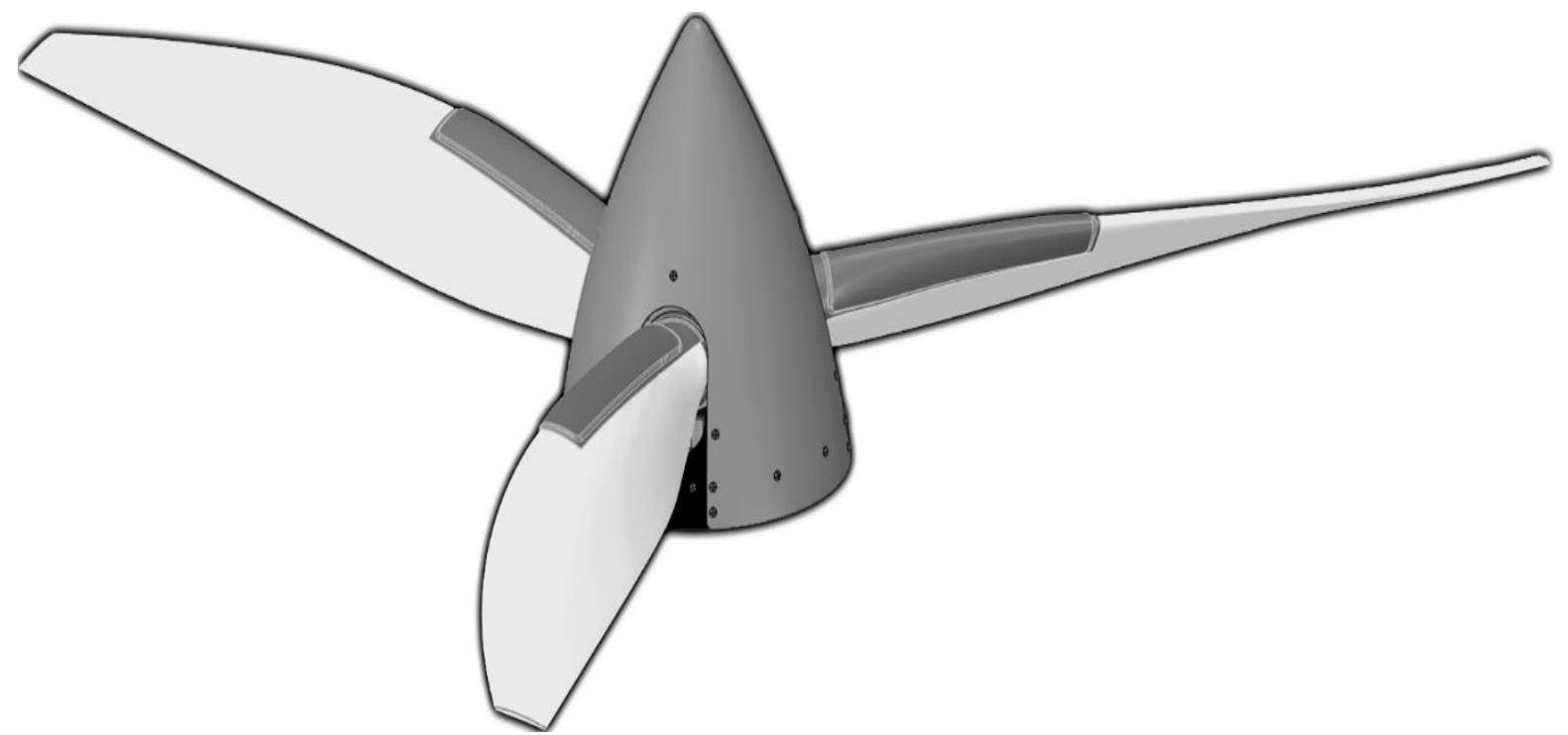

Fig. 1: Three-blade altitude propeller

Since this material has a shape of thin sheets with orthotropic properties, then the strength properties of the blade are determined by the specifics of prepreg sheet laying against each other, namely the angles of the laid composite mono-layers and fiber overlapping in the mono-layer.

The strength characteristics of the blades were calculated at the maximum rotation speed of the propeller equal to $2127 \mathrm{rpm}$ at the height of 0 meters. In this mode, the loads on the blades are maximum possible.

The structural optimization of the propeller blade was made in the ANSYS software. The number of composite material layers, laying angles and fiber overlapping were chosen as optimization parameters with the weight limitation of one blade being equal to $4 \mathrm{~kg}$. The SFX module was used for carrying out the aerodynamic analysis. The Mechanical and ACP modules were used for stressstrain analysis (SSA) and for the formation of composite layers, respectively. Steady and unsteady calculations were made in order to determine the aerodynamic loads. The loads were transferred as pressure fields from the module of aerodynamics to the Mechanical module. Layers with different parameters were laid in the ACP module.

\section{Calculating Aerodynamic Loads on the Surface of the Blades}

In the modeling of aerodynamic loads, a qualitative blocked grid was built with hexagonal elements in the entire computational space. In order to specify satisfactorily the behavior of flow in the proximity of walls, the mesh elements were crowded to the boundaries of the propeller blades. Figure 2 shows the position of the mesh elements on the surface of the blades and the hub. The entire number of calculation domain elements was $15 \mathrm{~m}$.

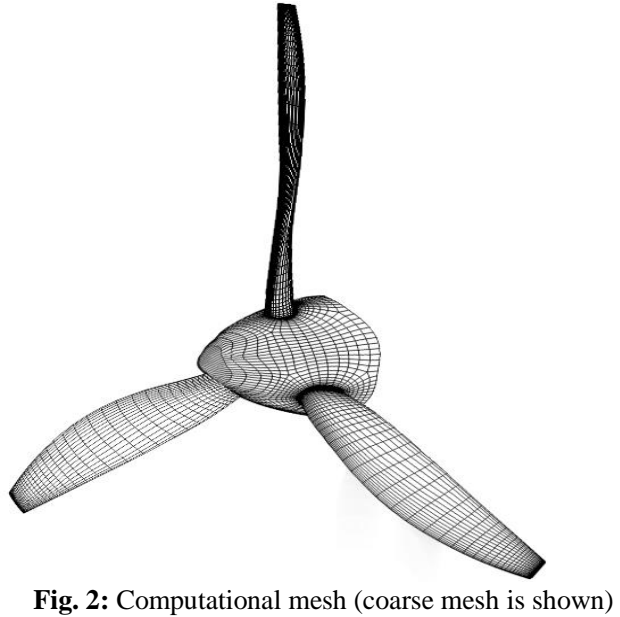

ANSYS CFX software was used to carry out the aerodynamic analysis of the propeller by a control volume method. Averaged Reynolds continuity, motion and energy conservation equations were solved together with closing equations of the SAS SST (The Scale-Adaptive Simulation Shear Stress Transport) turbulence model in case of an unsteady set-up. The SST (Shear Stress Transport) turbulence model was used in case of a steady set-up. The components of the velocity and pressure vector were connected with the use of a semi-implicit method for equations with pressure coupling - SIMPLE. The convergence of the problem was ensured by achieving the average squared disparities of the desired values to order $10^{-5}$.

The results of the aerodynamic analysis are presented as pressure fields on the surface of the blades in Fig. 3. The presented fields are conveyed as pressure values attached to the nodes of the finite element mesh and interpolated on the mesh for further SSA of the composite blade. 

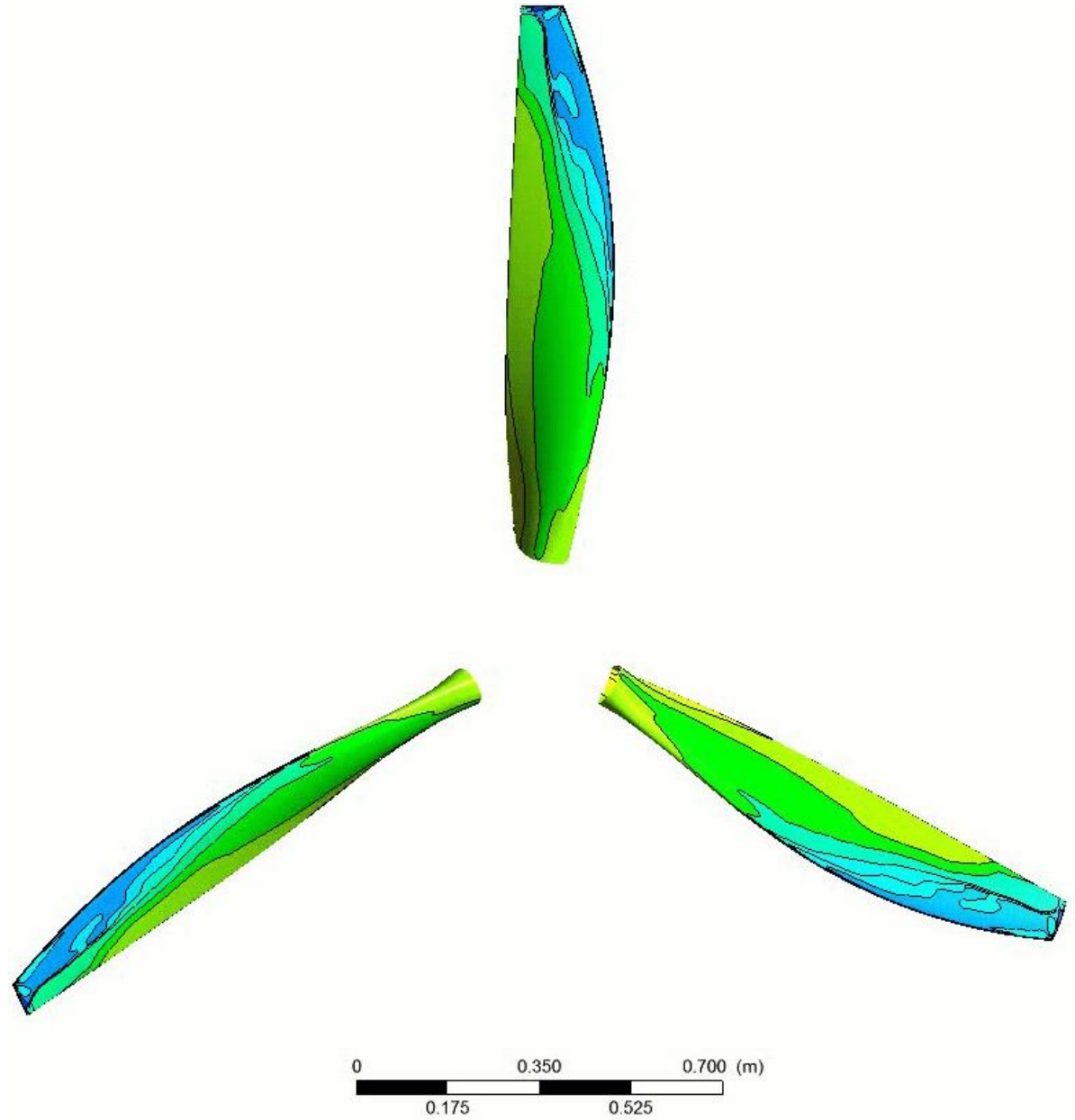

Fig. 3. Pressure distribution along the blade surface

\section{SSA of the Composite Blade}

During operation of the propeller, its blades are subject to big loads. The propeller is affected by the volumetric centrifugal force, which emerges due to the propeller rotation, the inertia forces at the starting of the propeller rotation and the distributed aerodynamic forces, which can change the shape of the blade significantly and, thus, alter the aerodynamic characteristics of the propeller a lot.

The body of the blade is a multilayer composite material with the density of $1780 \mathrm{~kg} / \mathrm{m}^{3}$. The materials of the blade were modeled with the parametrization of their physical properties. Two types of composites were researched with the fiber overlapping in the horizontal and vertical direction of two kinds: 50x50 and $75 \times 25$ percent. The orthotropic characteristics of the materials are presented in Table 1.

Table 1: Material properties

\begin{tabular}{|c|c|c|c|}
\hline & & $50 \times 50$ & $75 \times 25$ \\
\hline \multirow[t]{9}{*}{ Elastic characteristics } & $\mathrm{E}_{11}(\mathrm{hPa})$ & 60 & 85 \\
\hline & $\mathrm{E}_{22}(\mathrm{hPa})$ & 60 & 30 \\
\hline & $\mathrm{E}_{33}(\mathrm{hPa})$ & 6 & 8.5 \\
\hline & $\mathrm{G}_{12}(\mathrm{hPa})$ & 4 & 4 \\
\hline & $\mathrm{G}_{23}(\mathrm{hPa})$ & 0.615 & 0.615 \\
\hline & $\mathrm{G}_{13}(\mathrm{hPa})$ & 0.615 & 0.615 \\
\hline & $v_{12}$ & 0.054 & 0.085 \\
\hline & $v_{23}$ & 0.3 & 0.3 \\
\hline & $v_{13}$ & 0.3 & 0.3 \\
\hline \multicolumn{2}{|c|}{ Thickness of the monolayer, } & 0.2 & 0.14 \\
\hline
\end{tabular}

A finite element model of the blade was developed for the SSA. The above problem is reduced to a statistical problem of mechanics at the transition to a moving coordinate system at a constant speed of the blade rotation. The ANSYS Mechanical module was used for calculation and the ANSYS ACP module was used to model the composite. This type of analysis allows us to determine displacements, strains and internal forces in the body affected by various loads in the steady and unsteady set-up. Two way FSI calculation technology was used to transfer the loads [5].
The problem of mechanics was solved in a geometrically nonlinear set-up [6].

\section{Calculation Data}

According to the limitation system of the optimum problem, the blade weight cannot exceed $4 \mathrm{~kg}$ and the blade must be indestructible during operation of the propeller. 
The problem of the blade structural optimization includes two subproblems:

1. Determination of the optimum laying structure of the composite and its thickness along the height of the blade in order to ensure the required weight;

2. Statistical two way non-linear calculation of the composite blade affected by aerodynamic loads and centrifugal force to reveal the probability of the blade destruction.

In order to solve these problems, it is necessary to determine the structure of the composite, namely: the number of layers, the angles of material laying and the percentage ratio of fiber overlapping. To tackle this problem in ANSYS software, the angle of laying and the number of material layers, varied along the height of the blade, were set parametrically. The maximum displacement in the nodes of the finite element models at a set rotation of the propeller was chosen as a criterion. For the composite material with 50x50 overlapping and the thickness of one layer equal to $0.4 \mathrm{~mm}$, the results on the maximally possible deformation of the blade are presented in Table 2 .

Table 2: Maximum displacement for various angles of laying and various number of layers for the composite with 50x50 overlapping.

\begin{tabular}{|c|c|c|c|c|c|}
\hline \multirow{2}{*}{$\begin{array}{l}\text { Material } \\
\text { laying in } \\
\text { degrees }\end{array}$} & \multicolumn{5}{|c|}{ Maximum displacement, $\mathrm{mm}$} \\
\cline { 2 - 6 } & $\sim 2 \mathrm{~mm}$ & $\sim 3.2 \mathrm{~mm}$ & $\sim 4 \mathrm{~mm}$ & $\sim 5.2 \mathrm{~mm}$ & $\sim 6 \mathrm{~mm}$ \\
\hline $0^{\circ} 30^{\circ}$ & 19.89 & 19.78 & 19.61 & 19.42 & 19.31 \\
\hline $0^{\circ} 45^{\circ}$ & 18.89 & 18.74 & 18.59 & 18.42 & 18.31 \\
\hline $0^{\circ} 60^{\circ}$ & 16.39 & 16.21 & 15.82 & 15.67 & 15.44 \\
\hline $0^{\circ} 90^{\circ}$ & 16.73 & 16.49 & 16.31 & 16.14 & 15.96 \\
\hline
\end{tabular}

For the composite material with $75 \times 25$ overlapping and the thickness of one layer equal to $0.28 \mathrm{~mm}$, the results on the maximally possible deformation of the blade are presented in Table 3.

Table 3. Maximum displacement for various angles of laying and various number of layers for the composite with $75 \times 25$ overlapping.

\begin{tabular}{|c|c|c|c|c|c|}
\hline $\begin{array}{l}\text { Material } \\
\text { laying in } \\
\text { degrees }\end{array}$ & $\sim 2 \mathrm{~mm}$ & $\sim 3.2 \mathrm{~mm}$ & $\sim 4 \mathrm{~mm}$ & $\sim 5.2 \mathrm{~mm}$ & $\sim 6 \mathrm{~mm}$ \\
\cline { 2 - 6 } & & & & & 19.48 \\
\hline $0^{\circ} 30^{\circ}$ & 20.08 & 19.83 & 19.67 & 19.15 \\
\hline $0^{\circ} 45^{\circ}$ & 18.11 & 17.89 & 17.67 & 17.44 & 17.19 \\
\hline $0^{\circ} \mid 60^{\circ}$ & 16.59 & 16.37 & 16.19 & 15.94 & 15.76 \\
\hline $0^{\circ} \mid 90^{\circ}$ & 17.03 & 16.84 & 16.66 & 16.48 & 16.37 \\
\hline
\end{tabular}

It is shown that the composite having the laying angles of $0^{\circ} \mid 60^{\circ}$ and the number of layers equal to 13 (average thickness along the height of the blade is $\sim 5.2 \mathrm{~mm}$ with $50 \times 50$ overlapping) gives a lower maximum displacement, with the weight of the blade not exceeding $4 \mathrm{~kg}$. After determining the minimum displacements, two way FSI is calculated.

The aerodynamic loads obtained in the unsteady set-up are transferred to the ANSYS Mechanical module. These loads represent the information about the pressure on the blade surface. It should be noted that the change of pressure on the surfaces of the blades has a cyclic nature due to the unsteady processes of gas flowing around the blade.

The maximum deviation of the blade end edge from its initial position in case of centrifugal loading only is $\sim 20 \mathrm{~mm}$. In two way FSI analysis, additional aerodynamic loading produced a damping effect. Thus, the maximum displacement of the blade, due to the cyclic aerodynamic load, varied within a range of 7.34 to 8.24 $\mathrm{mm}$, which is much lower than $20 \mathrm{~mm}$ under centrifugal loading. Figure 4 shows the comparison of the deformed and non-deformed blade model. The displacements are scaled up. The maximum displacement of the blade position is insignificant and will not lead to blade destruction. It is worth mentioning that due to the cyclic nature of the load changes, it is reasonable to perform frequency analysis and fatigue life analysis to determine the operating life of the blade and the propeller as a whole.

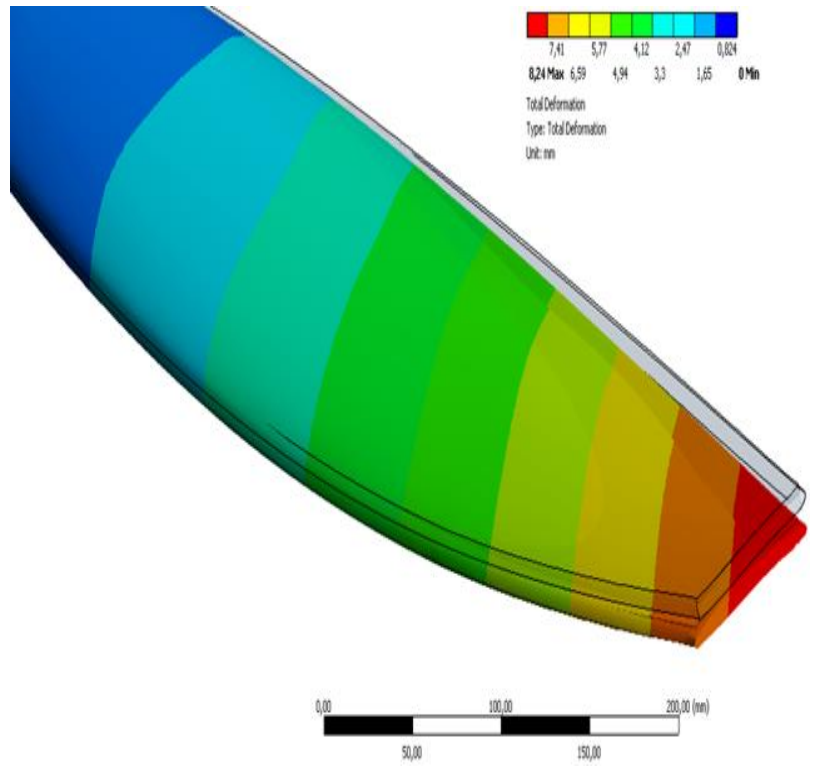

Figure 4. Comparison of a deformed and non-deformed blade model under the action of centrifugal and aerodynamic forces

\section{Conclusions}

A model was built which describes the behavior of propeller blades loaded centrifugally and aerodynamically. The structural optimization of the blade was made in terms of laying the composite materials by the thickness, angle and ratio of fiber overlapping in the composite material. It is shown that with the composite material laying technology of $0^{\circ} \mid 60^{\circ}$, it is possible to obtain minimum displacements and deflection of the blade end edges. Such laying increases the resistance of the blades to loads. As shown by the calculations, the biggest bending and torsional deformation will be observed with the laying of $0^{\circ} \mid 30^{\circ}$.

The obtained technique makes it possible to consider multiple effects on the blade and follow its deformation in dynamics. However, it should be considered that cyclically changing loads lead to the destruction of the blades. In further research, it is suggested that a mechanism for determining the fatigue life of the blades and proper frequencies be included into the calculation complex, apart from the coupled task.

It can be summarized that, in principle, the optimization problems of the type considered here refer to the problems of optimal control theory and represent a section of variational calculus for the problems with limitations in the form of differential equations in partial derivatives, i.e. the Lagrange - Boltz problem.

\section{Acknowledgment}

The work was financially supported by the Ministry of Science and Higher Education of the Russian Federation in the framework of the Federal Program "Research and Development in the Priority Areas of Development of the Russian Scientific and Technical Complex for 2014-2020", Activity 1.3., Subsidy Agreement No. 14.578.21.0244 of 26.09.2017, unique project identifier:

\section{RFMEFI57817X0244}

\section{References}

[1] Loytsyansky, L. G. (2003). Fluid mechanics. 7-th revised edition Moscow: Drofa, 812

[2] Skvortsov, Yu. V. (2013). Lecture notes for the discipline "Mechanics of Composite Materials. Samara: Samara State Aerospace University named after academician S.P. Korolyov, 94. 
[3] Glazunov, A. V. (2009). Turbulence vortex modeling with the use of combined dynamic closure: Part I. Problem statement, model specification and diagnostic numeric tests. The Bulletin of the Russian Academy of Sciences. Physics of atmosphere and ocean. 45(1). 7-28.

[4] Glazunov, A. V. (2009) Turbulence vortex modeling with the use of combined dynamic closure: Part II. Numerical experiments; Turbulence modeling in a channel with irregular boundaries, The Bulletin of the Russian Academy of Sciences. Physics of atmosphere and ocean. 45(1). 29-42.

[5] Schmucker, H., Flemming, F., \& Coulson, S. (2010). Two-way coupled fluid structure interaction simulation of a propeller turbine. International Journal of Fluid Machinery and Systems, 3(4), 342-351.

[6] Lurie, A. I. (1980). Non-linear theory of elasticity. Moscow: Science, 512.

[7] Boldyrev, Yu. Ya. (2016). Variational calculation and optimization methods. Textbook. SPb.: The publisher of the Polytechnic University, 240

[8] Hansen, M. O. (2015). Aerodynamics of wind turbines. Routledge.

[9] Meng, J., Hu, J., Xiao, H., \& Lv, M. (2017). Hierarchical optimization of the composite blade of a stratospheric airship propeller based on genetic algorithm. Structural and Multidisciplinary Optimization, 56(6), 1341-1352.

[10] Chen, F., Liu, L., Lan, X., Li, Q., Leng, J., \& Liu, Y. (2017). The study on the morphing composite propeller for marine vehicle. Part I: design and numerical analysis. Composite Structures, 168, 746757.

[11] Patel, Y., Gaurav, A., Srinivas, K., \& Singh, Y. (2017). A review on design and analysis of the propeller used in UAV. Int. J. Adv. Prod. Ind. Eng, 605, 20-23.

[12] Rigamonti, L., Forni, A., Sironi, M., Ponti, A., Ferretti, A. M. Baschieri, C., \& Pasini, A. (2018). Experimental and theoretical investigations on magneto-structural correlation in trinuclear copper (II) hydroxido propellers. Polyhedron, 145, 22-34.

[13] Lis, M. (2016). Numerical analysis and optimization of motoglider's propeller structure. Prace Instytutu Lotnictwa, 87-102.

[14] Suryanarayana, C., Rao, M. N., \& Raju, P. N. (2015). Structural Analysis of a Contra Rotating Propeller by using Finite Element Method (FEM). International journal of innovative research and development, 4(7)

[15] Li, W., Zhou, H., Liu, H., Lin, Y., \& Xu, Q. (2016). Review on the blade design technologies of tidal current turbine. Renewable and Sustainable Energy Reviews, 63, 414-422. 